\title{
Quantifying Global Uncertainties in a Simple Microwave Rainfall Algorithm
}

\author{
Christian Kummerow, Wesley Berg, Jody Thomas-Stahle, and Hirohiko Masunaga \\ Department of Atmospheric Science, Colorado State University, Fort Collins, Colorado
}

(Manuscript received 27 October 2004, in final form 14 April 2005)

\begin{abstract}
While a large number of methods exist in the literature for retrieving rainfall from passive microwave brightness temperatures, little has been written about the quantitative assessment of the expected uncertainties in these rainfall products at various time and space scales. The latter is the result of two factors: sparse validation sites over most of the world's oceans, and algorithm sensitivities to rainfall regimes that cause inconsistencies against validation data collected at different locations. To make progress in this area, a simple probabilistic algorithm is developed. The algorithm uses an a priori database constructed from the Tropical Rainfall Measuring Mission (TRMM) radar data coupled with radiative transfer computations. Unlike efforts designed to improve rainfall products, this algorithm takes a step backward in order to focus on uncertainties. In addition to inversion uncertainties, the construction of the algorithm allows errors resulting from incorrect databases, incomplete databases, and time- and space-varying databases to be examined. These are quantified. Results show that the simple algorithm reduces errors introduced by imperfect knowledge of precipitation radar (PR) rain by a factor of 4 relative to an algorithm that is tuned to the PR rainfall. Database completeness does not introduce any additional uncertainty at the global scale, while climatologically distinct space/time domains add approximately $25 \%$ uncertainty that cannot be detected by a radiometer alone. Of this value, $20 \%$ is attributed to changes in cloud morphology and microphysics, while $5 \%$ is a result of changes in the rain/no-rain thresholds. All but $2 \%-3 \%$ of this variability can be accounted for by considering the implicit assumptions in the algorithm. Additional uncertainties introduced by the details of the algorithm formulation are not quantified in this study because of the need for independent measurements that are beyond the scope of this paper. A validation strategy for these errors is outlined.
\end{abstract}

\section{Introduction}

Passive microwave algorithms designed to measure precipitation can be grouped into a relatively small number of classes depending upon the physical principle employed. The "emission" principle exploits the increased radiation originating from cloud and rainwater over radiometrically cold surfaces, such as the ocean. A well-known example using 19-GHz brightness temperatures is described by Wilheit et al. (1991) and later by Chang et al. (1999). While conceptually simple, emission techniques require knowledge of the freezing level, the integrated cloud and water vapor content, and the shape of the rainfall profile in order to relate the integrated water observed by the radiometer to the sur-

Corresponding author address: Christian Kummerow, Dept. of Atmospheric Science, Colorado State University, Fort Collins, CO 80523-1371.

E-mail: kummerow@atmos.colostate.edu face rainfall. In addition, these techniques must account for the rainfall inhomogeneity within rather large satellite fields of view $[\sim 50 \mathrm{~km}$ for the Special Sensor Microwave Imager (SSM/I) and $\sim 25 \mathrm{~km}$ for the Tropical Rainfall Measuring Mission (TRMM) Microwave Imager (TMI) and the Advanced Microwave Sounding Radiometer (AMSR)-E] in order to properly compensate for nonlinearities in the brightness temperature (Tb) versus water content relationships. The lack of constraints on these parameters translates directly into large uncertainties within individual pixels.

To reduce uncertainties at the pixel level, algorithms such as those developed by Petty (1994) and Aonashi and Liu (2000) rely on an approach similar to that above mentioned, but with additional frequencies used to help constrain the problem. The additional channels, unfortunately, do not provide any additional direct information about the surface rainfall. Instead, the additional channels are used to help describe some property of the underlying cloud, such as its convective or strati- 


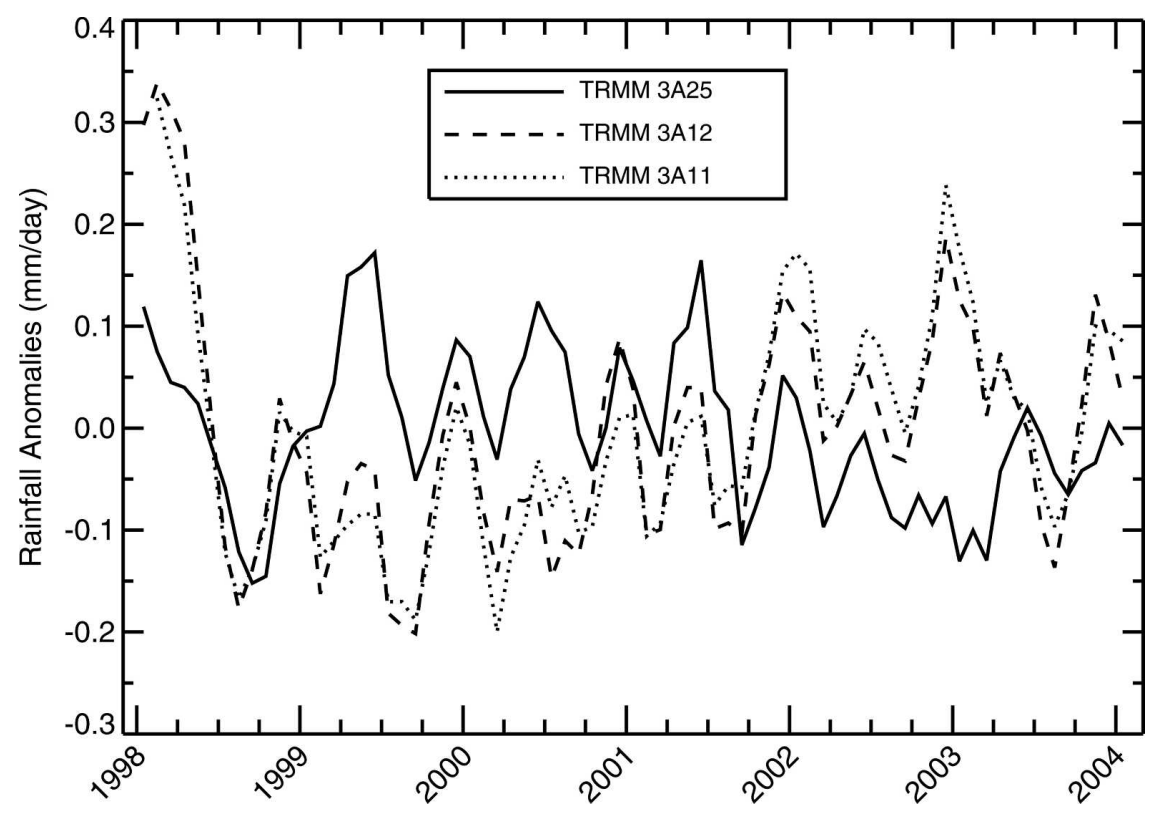

FIG. 1. Time series of TRMM operational radar (3A25) and radiometer rainfall anomalies. The radiometer products consist of the profiling algorithm GPROF (3A12) and the emission algorithm (3A11). Time series corresponds to oceanic rainfall between $36^{\circ} \mathrm{N}$ and $36^{\circ} \mathrm{S}$.

form nature or whether the scene corresponds to deep or shallow convection. This information is then used, in conjunction with appropriate parameterizations, to better constrain what the vertical rain profile or its horizontal inhomogeneity might be. As such, these methods can reduce the uncertainty at the pixel level, but only at the expense of additional assumptions and parameterizations that must themselves be validated if a complete error model is to be generated.

An even more complex family of algorithms is the Bayesian approaches that employ cloud resolving models (CRMs). These algorithms are described by a number of authors (e.g., Mugnai et al. 1993; Kummerow and Giglio 1994; Smith et al. 1994; Marzano et al. 1999; Bauer 2001; Kummerow et al. 2001). In these methods, the CRM outputs are coupled with radiative transfer calculations to construct a priori databases of satellite observables along with the cloud model hydrometeor fields. A Bayesian or probabilistic approach is then used to compute the most likely a posteriori distribution when constrained by the actual measurements. Unlike the previous multifrequency approaches, these schemes can make direct use of explicit physical mechanisms contained in the CRMs to link satellite observables to the rain structure. The disadvantage of these approaches is their susceptibility to errors in the a priori database in terms of the accuracy of the microphysical details provided by the CRM (e.g., Panegrossi et al. 1998), the completeness of the CRM databases, and the fidelity with which the model outputs capture differences in climate regimes. These climate regime biases can be difficult to observe at individual validation sites because of the large random noise associated with individual clouds. They are, however, critical to understanding climate signals and trends.

One apparent manifestation of these biases is the systematic difference in the retrieved rainfall products from the TRMM radar and radiometers during El Niño-Southern Oscillation (ENSO) periods (Berg et al. 2002). During ENSO both the emission scheme (TRMM 3A11; Wilheit et al. 1991) and the cloud model-based Bayesian scheme (TRMM 2A12; described in Kummerow et al. 2001) show marked increases in tropical oceanic precipitation that are not seen by the TRMM radar (Robertson et al. 2003). It is unlikely that the differences are the result of simple radiometer algorithm artifacts because both the "emission"-based algorithm and the Bayesian schemes show amazingly consistent climate rainfall trends despite the different formulations and large differences at individual storm scales. Figure 1 shows a time series of the two TRMM radiometric algorithms and the TRMM radar (2A25) rainfall for tropical oceans.

Figure 1, in addition to revealing the rainfall trends observed by the TRMM radar and radiometer algorithms, also highlights the difficulties associated with validating rainfall products. The average oceanic rainfall from the two radiometeric algorithms shown in Fig. 
1 differs by only $0.06 \mathrm{~mm}$ day $^{-1}$. This corresponds to roughly $4 \%$ of their mean values. The difference between radar and radiometer rainfall during the ENSO event of $1997 / 98$ is only $7 \%$ of the average mean global rainfall. At the global scale, represented by Fig. 1, these $4 \%-7 \%$ differences can be quite significant. These same differences, however, are much smaller than the $10 \%-15 \%$ regional differences known to exist between these products and are still smaller than most direct comparisons between satellite- and ground-based observations.

Despite the above difficulties related to the validation of rainfall products, it is clear that algorithms designed for the future must be able to fully characterize uncertainties at any space and time scale being considered by the users. This ranges from instantaneous estimates needed for many hydrologic and weather forecasting applications to large space and time averages required for climate model verification and climate trend monitoring. While the need for a full description of uncertainties is perhaps self-evident, such a complete error characterization does not currently exist. This paper describes an algorithm designed to quantify these both instantaneous as well as systematic errors at longer time and space scales. To focus on errors, the algorithm itself has been kept extraordinarily simple, and as such is not intended to replace any of the operational rainfall algorithms. For the time being, the algorithm is also limited to oceanic areas.

With these simplifications, one may legitimately ask whether such a scheme is appropriate to understand uncertainties in the more complex algorithms, such as the Goddard profiling algorithm (GPROF). While there is certainly some information that can be gleaned from this study, it is premature to answer this question fully. The true answer, however, may not lie in developing error models to fully account for all error sources in the already complex algorithms, but may lie equally in a simpler algorithm such as the one described here, whose sophistication is only increased when an error model can be simultaneously constructed.

\section{Algorithm framework}

Conceptually, all physically based algorithms begin by computing radiances that would be observed from a given cloud structure. These theoretically computed radiances $(\mathrm{Tb})$ are then inverted to obtain the surface rainfall corresponding to an observed radiance field. Unfortunately, this first step is already fraught with difficulties because a typical rainfall cloud has many more free parameters than can reasonably be modeled, let alone retrieved. Additional constraints are needed. The different methods used to introduce these constraints, or a priori information, effectively describe the algorithms discussed in the previous section. Nonetheless, one can write the forward problem in symbolic form,

$$
\mathrm{Tb}=f\left(r, v_{i}, c_{i}\right),
$$

where $\mathrm{Tb}$ can be a single brightness temperature or combination of channels, $r$ is the surface rainfall that is being retrieved, $v_{i}$ is cloud and environmental parameters that one explicitly allows to vary in the forward model, and $c_{i}$ is the cloud and environmental parameters that one chooses to keep fixed. One can also have parameterizations, such as coupling the vertical liquid water profile to the surface rainfall, but insofar as such a parameterization is explicit, only the rainfall rate would be considered a variable in this symbolic notation.

Within this framework, it would appear logical to choose, in addition to the surface rainfall, parameters that strongly affect the $\mathrm{Tb}$, as do the variables in Eq. (1), while keeping the remaining parameters constant. This is done in the Wilheit et al. (1991) scheme. The freezing level is an assumed variable because of its strong effect on determining the column-integrated water and thus upwelling Tb. All other variables are kept fixed or are parameterized as a function of the surface rainfall and freezing level. The main advantage of the scheme is its simplicity. This simplicity, however, also implies that the algorithm has no mechanism to account for different storm structures that may be observed.

In contrast to this simple scheme described above, GPROF (Kummerow et al. 2001) uses CRMs to construct a database of a priori precipitating clouds that may be seen by the satellite. In this case, because each profile is a unique entity with all parameters described by the CRM, the profile itself must be considered a variable. The specific characteristics of each profile represent the constant terms in Eq. (1). The main problem with this scheme is that there is no guarantee that the variables (CRM profiles) fully characterize the observed space of profiles and that the constant terms (the profile characteristics) are correct. The first problem is generally referred to as the completeness problem. The latter is related to errors in the CRMs themselves and can be referred to as the correctness problem. In short, the GPROF scheme avoids the lack of flexibility that is inherent to the simple emission schemes. In exchange, however, it introduces two assumptions- the completeness and the correctness of the CRM profiles-that are both very difficult to validate. 


\section{The error algorithm}

The error algorithm described here is a hybrid between the simple emission scheme described by Wilheit et al. (1991) and GPROF, which is a cloud model-based Bayesian scheme described by Kummerow et al. (2001). It uses the simple physical relation between observed increases in the $19-\mathrm{GHz} \mathrm{Tb}$, but in the statistical framework of GPROF in order to take advantage of the simple uncertainty model afforded by that scheme. Unlike GPROF, however, the a priori database is constructed directly from the TRMM precipitation radar (PR) rainfall product (Iguchi et al. 2000), version 5. To the extent that the TRMM PR captures spatial and temporal changes in cloud properties, these variations in the a priori database can be explicitly examined. Experiments described in section 3 demonstrate that this procedure also eliminates the "completeness" problem. The "correctness" problem is not eliminated by this a priori database, but a careful analysis of potential errors in the TRMM PR products used to construct the database allows these errors to be quantified.

Construction of the a priori database begins by identifying $7 \times 11$ TRMM PR pixels that fully encompass the 19-GHz field of view (FOV) corresponding to the 51st and 54th TMI pixels along each scan line. These pixels were selected in order to remain near the center of the scan (where TRMM PR vertical resolution is optimal), while avoiding the excessive overlap of the 52nd and 53rd pixel positions. A mean brightband height is identified within each TMI FOV by averaging brightband height information provided by the PR pixels within the 19-GHz FOV. If no PR pixel contains a bright band, the search area is expanded successively to $17 \times 21,27 \times 31$, and finally $49 \times 51 \mathrm{PR}$ pixels. If no bright band is found, a climatological brightband height is assigned to these pixels based upon the observed sea surface temperature. Only $7 \%$ of the rain falls in this category.

The vertical rain profile is taken from the TRMM PR standard rainfall product. The rain rate is considered liquid from the surface to three layers $(750 \mathrm{~m})$ below the layer containing the bright band, while snow is considered from the top of the reflectivity profile to one layer $(250 \mathrm{~m})$ above the layer in which the bright band was found. These definitions are consistent with the TRMM PR rain algorithm. Between the rain and snow layers, there are four TRMM PR layers that may contain the bright band itself. The TRMM PR rainfall in these layers is not used because they may be contaminated by the bright band itself. Instead, this region is filled by linearly interpolating the liquid and snow- water content within the melting layer. No partially melted particles are considered.

The relations needed to convert rain rate to liquid and snow-water content from the TRMM PR rainfall product can be reconstructed from relations presented by Iguchi et al. (2000) and Masunaga et al. (2002). Instead of the five separate levels described in those studies, only the lowest level (for liquid) and highest level (for snow) discussed in those studies are used here. Specifically, the relations for convective and stratiform rain (as defined in the standard TRMM PR products) are $M_{\text {conv,liq. }}=0.074 R^{0.87}, M_{\text {strat,liq. }}=0.064 R^{0.89}$, $M_{\text {conv,snow }}=0.217 R^{0.91}$, and $M_{\text {strat,snow }}=0.196 R^{0.92}$. While the operational TRMM PR algorithm adjusts these relationships when the path-integrated attenuation (PIA) derived from the PR is deemed reliable, this adjustment is not done here in order to keep the relations as simple as possible. The "others" category defined by the TRMM PR algorithm has the same coefficients as the convective rain for these two levels and is thus treated as convection. The above technique places hydrometeors in 250-m layers in each of the $7 \times 11$ columns of the TRMM PR. For computational efficiency, and because of the lack of sensitivity of the $19-\mathrm{GHz}$ radiances to small ice, the hydrometeor profiles are only extended to 40 layers $(10 \mathrm{~km})$ above the surface.

In addition to the hydrometeor concentrations, surface conditions as well as any cloud water and water vapor must be specified in order to simulate the upwelling radiances. The sea surface temperature (SST) was taken from the 3-day-averaged SST available from Remote Sensing Systems (see information online at http://www.ssmi.com). Wind speed was also obtained from the 3-day-averaged surface wind computed by Remote Sensing Systems. While the wind field is not directly related to the wind during the time of the precipitation event, it should provide a realistic distribution of surface winds. The remaining parameters needed for the atmospheric model are all assumed constant and are supplied by the algorithm. The temperature profile is assumed to follow a constant lapse rate of $6 \mathrm{~K} \mathrm{~km}^{-1}$ using the layer above the bright band [freezing level $(\mathrm{FL})]$ as the $0^{\circ} \mathrm{C}$ isotherm. Pressure is computed using the hydrostatic equation with the surface pressure set at $1013 \mathrm{mb}$. Cloud water and water vapor are assigned based upon the pixels' rain status. If the pixel is raining, $0.5 \mathrm{~kg} \mathrm{~m}^{-2}$ of cloud water is assigned to the pixel. Following Wilheit (1986), the cloud water is distributed in the two layers $(500 \mathrm{~m})$ below the freezing level. If the pixel is not raining, but is adjacent to rain, $0.2 \mathrm{~kg} \mathrm{~m}^{-2}$ of cloud water are assigned and distributed as before. The remaining pixels are assigned no cloud 


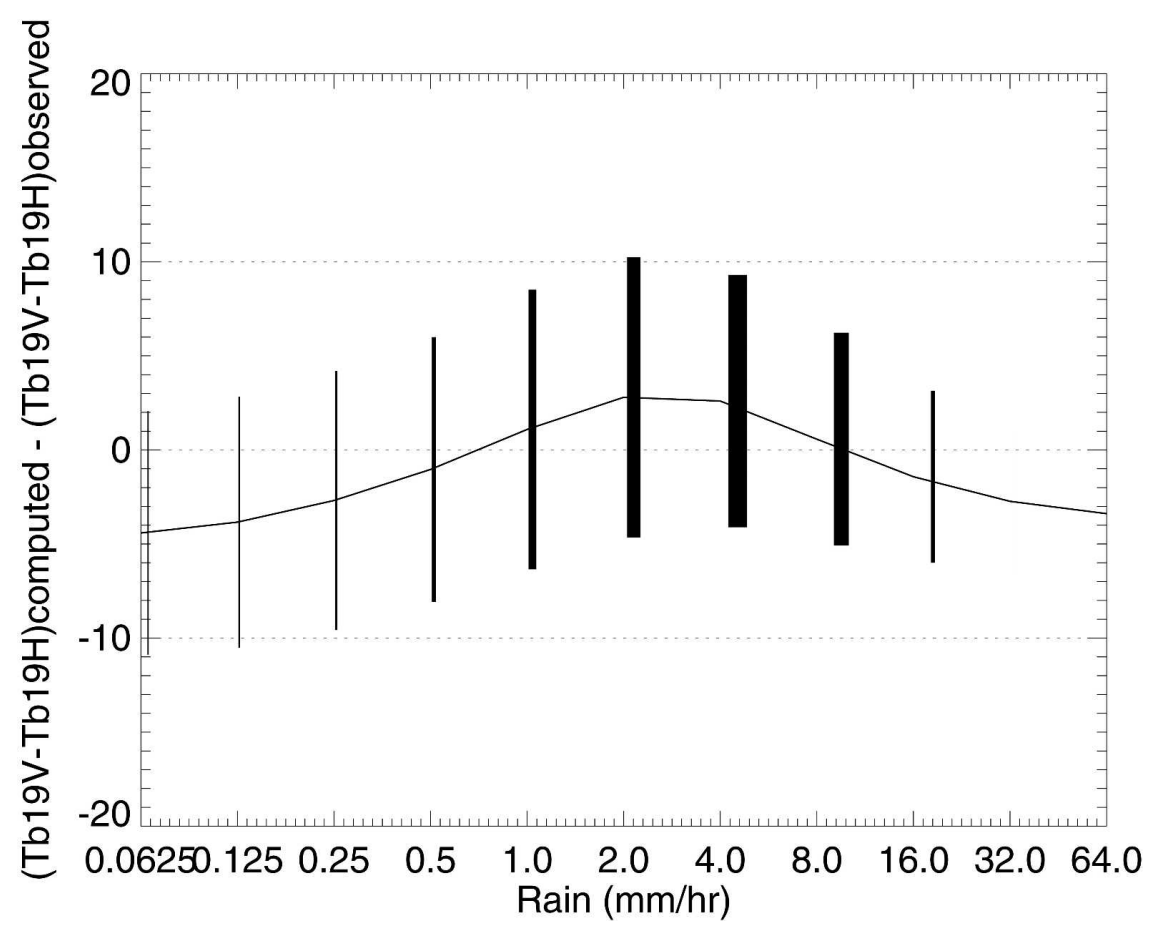

FIG. 2. Statistics of computed minus observed $\mathrm{Tb}$, defined as $\mathrm{Tb}_{19 \mathrm{~V}}-\mathrm{Tb}_{19 \mathrm{H}}$, for all database pixels created from Dec 1999 data. The height of the vertical bars corresponds to the standard deviation about the mean, while the width of the bars represents the relative contribution of each rain rate interval to the total rain. The rainfall for each pixel is taken to be the TRMM PR surface rainfall.

water. The relative humidity follows the cloud water scheme. It is assumed $80 \%$ at the surface, increasing to $100 \%$ in the cloud layer and then decreasing $10 \% \mathrm{~km}^{-1}$ above the freezing level. When no clouds are present, the relative humidity is kept constant at $80 \%$ up to the freezing level and then decreases by $10 \% \mathrm{~km}^{-1}$ as with the raining pixels.

The above profiles (at TRMM PR spatial resolution) are used to compute brightness temperatures using the Eddington approximation (Kummerow 1993). In the Eddington solution, diffuse radiances are computed first. Plane-parallel theory is used for this. The planeparallel diffuse radiance is then used in conjunction with a ray-tracing method to account for the actual geometry of the cloud. This solution is generally referred to as the slant-path approximation. While not exact, the technique captures the horizontal variability quite well (Roberti et al. 1994). The Tbs computed at the TRMM PR resolution $(\sim 4 \mathrm{~km})$ are then convolved with the TMI antenna gain functions to obtain the TMI $19-\mathrm{GHz}$ computed brightness temperatures. In a slight deviation from the Wilheit et al. (1991) scheme, the current algorithm uses the $\mathrm{Tb}$ difference at $19 \mathrm{GHz}$ (i.e., $\mathrm{Tb}=$ $\left.\mathrm{Tb}_{19 \mathrm{~V}}-\mathrm{Tb}_{19 \mathrm{H}}\right)$ to ensure that the function is always monotonic.
Differences between observed and computed Tbs can now be compared and are shown in Fig. 2 as a function of rainfall rate (as given by the TRMM PR). Ideally, Fig. 2 should be a horizontal line with zero bias and small random variations. This, however, would only be true if all of the assumptions stated earlier are correct and the TRMM PR rainfall is fully consistent with TMI observations. This is clearly not the case, with deviations up to $5 \mathrm{~K}$ for the lightest rain-rate bin. For low FOV-averaged rainfall rates (less than $0.25 \mathrm{~mm}$ $\mathrm{h}^{-1}$ ), TRMM PR rainfall is not sufficient to significantly alter the $\mathrm{Tb}$. The cloud water and water vapor assumptions appear to lead to excess attenuation that in turn leads to lower computed than observed Tb. Differences could also be attributed to a bias in the wind speed that must be interpolated from nearby rain-free pixels. At moderate rainfall rates $\left(0.25-4 \mathrm{~mm} \mathrm{~h}^{-1}\right)$, the observed $\mathrm{Tb}$ indicate more attenuation than the computed $\mathrm{Tb}$. This could be a result of insufficient cloud water and water vapor, or the result of neglecting melting hydrometeors. It could also be attributed to an underestimation of rainfall by the TRMM radar. The bias reverses again for very high $\mathrm{FOV}$-averaged rainfall $\left(>32 \mathrm{~mm} \mathrm{~h}^{-1}\right)$, which could be because of an overestimation of rainfall by the TRMM PR but may also be 
caused by some ice scattering effects at these high rainfall rates.

Assumptions could be changed to achieve better agreement between computed and observed Tbs. Because there are virtually no data to constrain the assumptions, however, they were not modified and the a priori database was constructed with the stated assumptions for nonraining parameters as well as TRMM PR rainfall. Three months, December 1999, January 2000, and February 2000 (henceforth called DJF 1999-2000), were used to construct the a priori database used for most of this study. To look at temporal changes, two additional databases covering DJF 1997-98 and DJF 1998-99 were constructed to contrast the weak La Niña year (1999-2000) with strong El Niño (1997-98) and La Niña (1998-99) years. Using only two TMI pixels per scan, each DJF period generates approximately 650000 raining profiles for use in the database.

In addition to the raining pixels in the database, a second database is created to determine the probability of rain for each pixel as a function of $\mathrm{Tb}$ and SST. For simplicity, the present scheme uses observed $\mathrm{Tb}$ in conjunction with TRMM PR-determined rain/no-rain information to construct this database with $1-\mathrm{K}$ intervals in both $\mathrm{Tb}$ and SST.

The retrieval algorithm itself is straightforward. For each observed $\mathrm{Tb}$ and SST, the rain/no-rain database is searched first to establish the probability of whether the pixel is raining. If rain is possible, the raining database is searched to find all entries having $\mathrm{Tb}$ and SST within 2.2 and $3 \mathrm{~K}$, respectively. All entries within this interval are given equal weight in the final solution. The $2.2-\mathrm{K}$ uncertainty in $\mathrm{Tb}$ is made up of an uncertainty of $1 \mathrm{~K}$ for the sensor noise and $2 \mathrm{~K}$ for uncertainties in the radiative transfer computations. The latter is consistent with radiative transfer uncertainties at $19 \mathrm{GHz}$ presented in Smith et al. (2002). A 3-K uncertainty was used for SST because it comes from a 3-day average rather than the pixel itself. Results showed very little sensitivity if uncertainties were changed by $\pm 1 \mathrm{~K}$ for either parameter.

Figures $3 \mathrm{a}$ and $3 \mathrm{~b}$ compare the resulting rainfall accumulations from the above algorithm for December 1999 with the latest operational version of the GPROF algorithm [version 6 (V6)] being utilized by the TRMM project. Both rainfall and rain/no-rain databases from the same time period were used in the retrieval. The corresponding zonal mean rain accumulations are shown in Fig. 3c. As can be seen, there is generally good agreement in the Tropics but the error algorithm produces more rain outside of $30^{\circ}$ latitude, particularly in the Southern Hemisphere. This leads to somewhat greater overall accumulations $\left(2.76 \mathrm{~mm} \mathrm{day}^{-1}\right.$ for the error algorithm versus $2.60 \mathrm{~mm}$ day $^{-1}$ for GPROF V6).

This example is used merely to illustrate that reasonable monthly rainfall accumulations can be obtained from this simple $19-\mathrm{GHz}$ emission algorithm. Little weight should be given to the overall agreement with the operational algorithm because the absolute magnitude of the error algorithm can be modified through the somewhat arbitrary assumptions related to cloud water, water vapor, the melting level, and drop size distribution (DSD) assumptions detailed in the previous section.

\section{Algorithm uncertainties}

The motivation of the above algorithm, as stated earlier, was not to develop yet another rainfall product, but to instead develop a simple algorithm that is amenable to the development of a complete error model. Given the complexities of such an error model, a simple algorithm is convenient. To that end, the probabilistic methodology selected for the algorithm can immediately be used to derive the uncertainty related to the inversion methodology. Statistics, which reflect the variability of solutions found within the $2.2-\mathrm{K}$ rms specified in the retrieval as a function of the retrieved surface rainfall, are presented in Fig. 4. The rainfall is again based on December 1999 TMI data. For this figure, the 3-month database for DJF 1999-2000 was used. The rain rate is the conditional rain rate. The probability of rain for each rain-rate interval is therefore also presented.

Uncertainties, as a percent of rainfall, are generally greatest for low rainfall rates as seen in Fig. 4. At these light rainfall rates, the $\mathrm{Tb}$ signal at $19 \mathrm{GHz}$ is very weak and can be confused with uncertainty in the observed $\mathrm{Tb}$ or SST. Minimum uncertainties are found in the 2-10 $\mathrm{mm} \mathrm{h}^{-1}$ range. Above $10 \mathrm{~mm} \mathrm{~h}^{-1}$, uncertainties again increase because of saturation of the $\mathrm{Tb}$ signal. Relatively little rain $(<18 \%)$ falls at this high rain-rate category. These results are consistent with the random errors reported by L'Ecuyer and Stephens (2002), for the TRMM operational algorithm.

The inversion uncertainties are easily computed in a statistical algorithm framework. They are, unfortunately, not the only error sources. Errors in the a priori database itself cannot be ignored. These errors result when the a priori database is either not correct, not complete, or contains spatial and/or temporal variability that cannot be resolved by the algorithm itself. These sources of uncertainty are treated individually in the following subsections. The final source of uncertainty, addressed in section $3 \mathrm{~d}$, is the result of algorithm formulation errors. This category consists of any errors 
a TRMIM GPROF (2A12), vel'sion 6

$\langle R\rangle=2.60$ min lay $^{-1}$

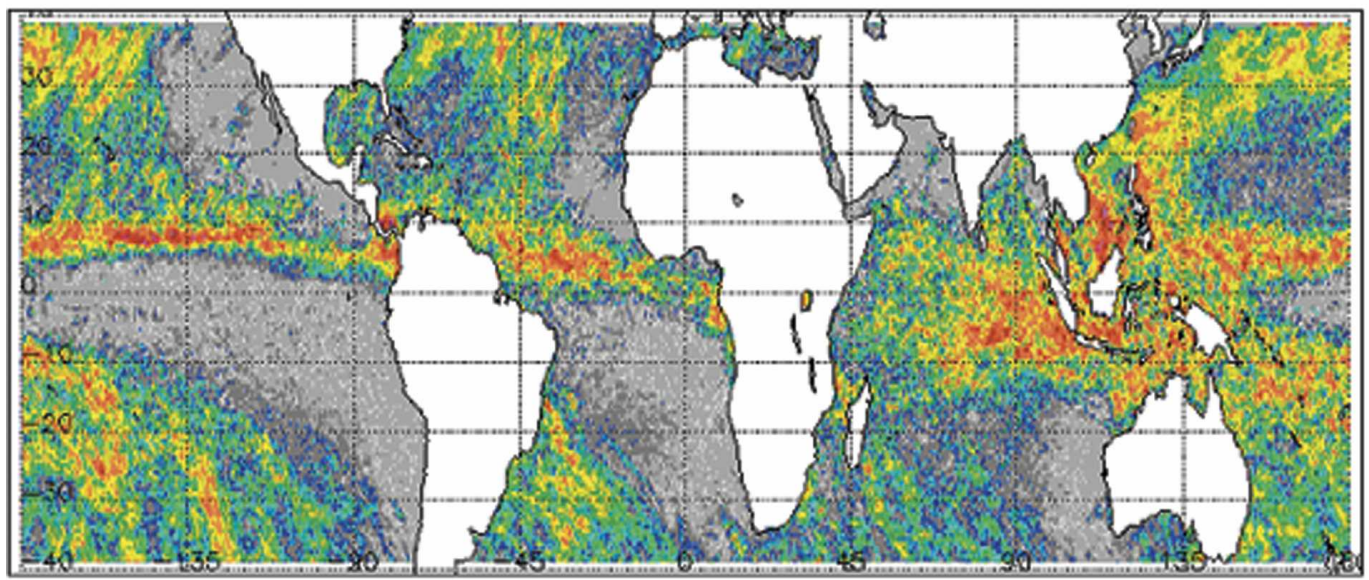

$\mathrm{b}$

Erroor Algorithm

$\langle R\rangle=2.76 \mathrm{~mm}^{\text {day }}{ }^{-1}$
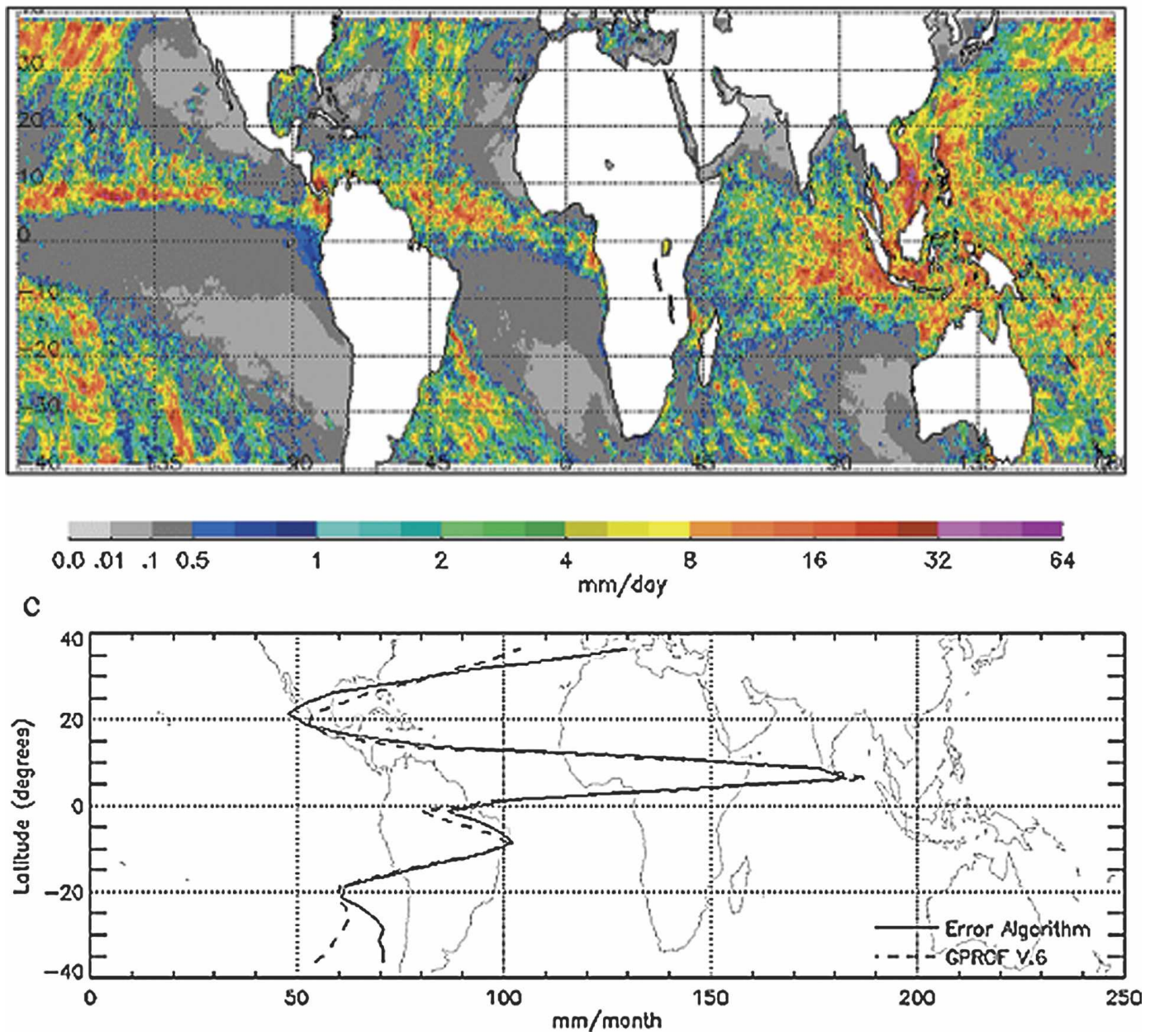

FIG. 3. Comparison between (a) the TRMM operational GPROF radiometer product (3A12), and (b) the current error algorithm applied to Dec 1999 with the DJF 1999-2000 database. (c) Zonal mean rain accumulations for the two products. 


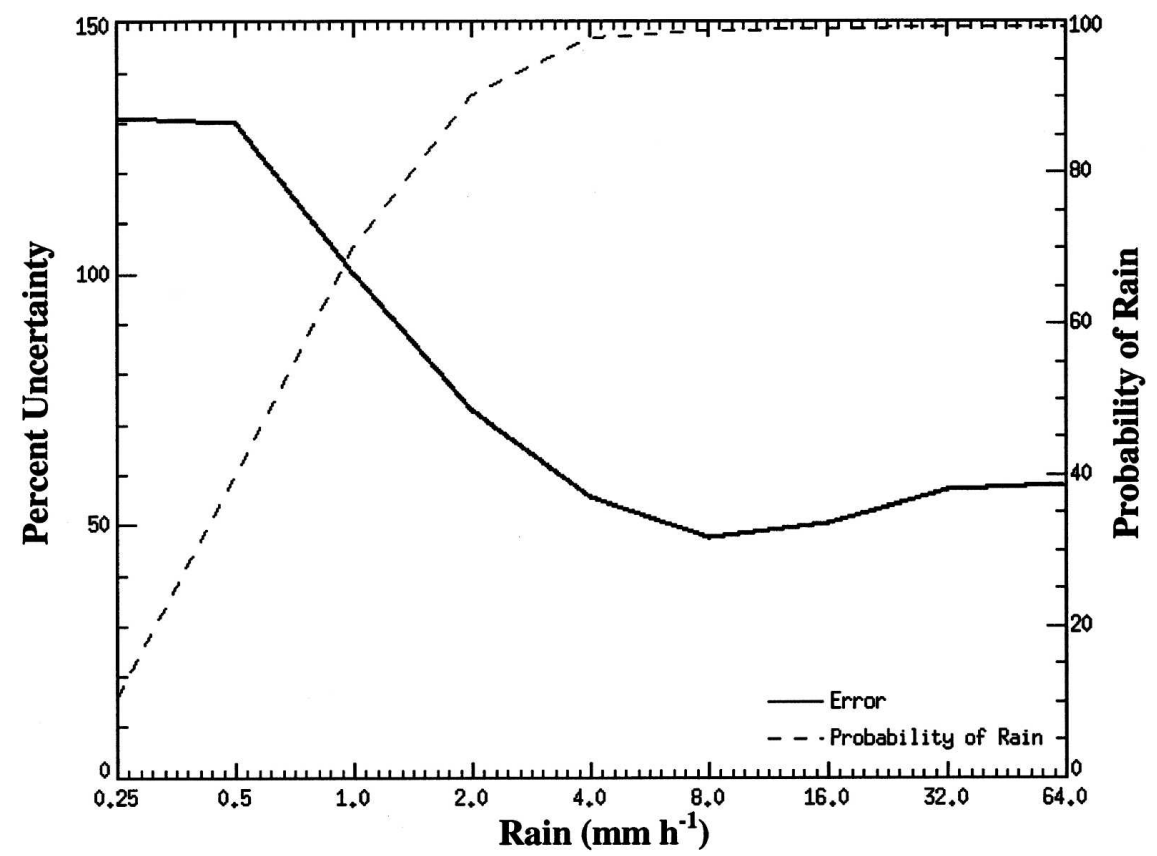

FIG. 4. Inversion uncertainties related to the nonuniqueness in the Tb to rainfall relation. The uncertainties are conditional upon the pixel having rain. The average probability is also shown between $0 \%$ and $100 \%$, with values labeled on the right side of the plot.

that could exist in the code itself, any errors in the assignment of assumed parameters discussed in section 2 , as well as the constant formulation of parameters that may have large space/time variability in nature. These cannot be quantified by the satellite and require independent validation data. While this is beyond the scope of this paper, a discussion aimed at defining an approach to evaluating these potential errors is nonetheless included in section $3 \mathrm{~d}$.

\section{a. Correctness of the a priori database}

The TRMM PR operational rainfall product (2A25, version 5) was used to construct rainfall profiles. These, along with some constant parameters defined in section 2 , plus radiative transfer computation, were used to create the a priori database. The constant parameters described in section 2 are intended to represent mean assumptions. Here, the uncertainties introduced by imperfect TRMM PR data are examined.

Ideally, one would run the TRMM PR operational algorithm with various DSD assumptions to assess the extent to which the mean TRMM PR rainfall results are uncertain. Because of the complexity of the operational TRMM PR algorithm, however, this proved unfeasible. Instead, this section examines the sensitivity of the current retrieval algorithm to systematic changes in the TRMM PR output. Because any errors in the shape of the rain profile must be coupled to the integrated liquid water retrieved by the TRMM PR through the DSD assumption, however, sensitivity tests were only conducted to quantify the changes in the retrieved rainfall as a function of changes in the integrated TRMM PR rainwater content. To first order, this is intended to represent changes in the retrieved profile if DSDs could be changed. This procedure, while far from perfect, is nonetheless illustrative for this first attempt at constructing a complete error model. December 1999 was used to construct the baseline and modified databases used in these experiments. This represents approximately 220000 raining pixels. December 1999 was also used to assess the retrieval sensitivity.

For these experiments, the TRMM PR rainfall is modified throughout the rain column by a fixed percentage in the range of $\pm 20 \%$. This is a reasonable uncertainty in the global products dictated by the global mean energy budget uncertainties (Kiehl and Trenberth 1997). Table 1 lists the results from these experiments as a percentage of change from the baseline algorithm. If the percentage remains near $0 \%$, then there is no sensitivity to errors in the a priori rainfall rate prescribed by the TRMM PR. If the percentage follows the percentage change in the TRMM PR product, then the algorithm has no skill and simply reproduces any errors in the a priori database. The five rain-rate categories shown in Table 1 were selected to each contain 
TABLE 1. Net bias in retrieved rainfall resulting from specified error in the TRMM PR volumetric rainfall.

\begin{tabular}{|c|c|c|c|c|c|c|}
\hline & $\begin{array}{c}\text { Percent } \\
\text { rain }\end{array}$ & TRMM PR + 20\% & TRMM PR + 10\% & $\begin{array}{c}\text { TRMM } \\
\text { PR }\end{array}$ & TRMM PR - 10\% & TRMM PR $-20 \%$ \\
\hline$<1 \mathrm{~mm} \mathrm{~h}^{-1}$ & 20.3 & $+6.1 \%$ & $+3.2 \%$ & $0 \%$ & $-3.2 \%$ & $-6.6 \%$ \\
\hline $1-3 \mathrm{~mm} \mathrm{~h}^{-1}$ & 20.2 & $+3.7 \%$ & $+2.1 \%$ & $0 \%$ & $-2.4 \%$ & $-5.3 \%$ \\
\hline $3-6 \mathrm{~mm} \mathrm{~h}^{-1}$ & 22.7 & $+2.6 \%$ & $+1.1 \%$ & $0 \%$ & $-2.1 \%$ & $-3.5 \%$ \\
\hline $6-10 \mathrm{~mm} \mathrm{~h}^{-1}$ & 19.1 & $+3.9 \%$ & $+2.5 \%$ & $0 \%$ & $-2.9 \%$ & $-7.4 \%$ \\
\hline$>10 \mathrm{~mm} \mathrm{~h}^{-1}$ & 17.6 & $+6.1 \%$ & $+2.4 \%$ & $0 \%$ & $-0.3 \%$ & $+0.6 \%$ \\
\hline Total & 100.0 & $+4.3 \%$ & $+2.1 \%$ & $0 \%$ & $-2.1 \%$ & $-4.6 \%$ \\
\hline
\end{tabular}

approximately $20 \%$ of the total rainfall. The exact fractions are provided in the table.

Results from Table 1 indicate that the uncertainty in the radiometer algorithm is approximately $22 \%$ of the uncertainty in the TRMM PR rainfall, or $5 \%$ for an uncertainty of about $20 \%$ in the TRMM PR global mean rainfall. This uncertainty comes primarily from low rainfall rates where the $19-\mathrm{GHz}$ channels appear insensitive to actual changes in the rain. A future refinement of the algorithm could use higher frequencies as proposed by Hong et al. (1997) to reduce this sensitivity to uncertainties in light rainfall. Here, however, the aim is not to immediately improve the algorithm, but rather to explicitly quantify the uncertainties of the current formulation. A 5\% uncertainty is therefore used for database correctness issues.

\section{b. Completeness of the a priori database}

Incomplete databases have long been speculated to be a significant source of uncertainty in retrieval schemes relying on CRMs for their a priori databases. These cloud models are difficult to run and to date only a handful of state-of-the-art simulations are available for retrieval algorithms (Kummerow et al. 2001). Because the TRMM PR-generated databases can be made extremely large, this source of uncertainty can be investigated directly for the error algorithm considered here. Comparisons with progressively smaller database subsets suggests that results are stable to the $1 \%$ level down to approximately 2000 entries when compared to the benchmark database from DJF 1999-2000, containing 666713 entries.

An alternative approach to assessing database completeness is to evaluate the expected uncertainty resulting from a finite number of database entries that match a given set of observations. The best estimate for rainfall is given by the mean value of all database entries fitting the observations ( $\mathrm{Tb}$ and SST in the baseline error algorithm). The retrieval uncertainty, or standard deviation $\sigma_{\text {inversion, }}$, is simply the average deviation of the individual database entries fitting the observation vector. Likewise, the average uncertainty in the final solution, the standard deviation of the mean, is simply given by $\sigma_{\text {inversion }} /(n)^{1 / 2}$, where $n$ is the total number of database entries that match the observation vector. As a way of illustrating this result, a pixel with a $\mathrm{Tb}$ of $30 \mathrm{~K}$ and an SST of $300 \mathrm{~K}$ is considered. The uncertainty in the input variables is, as before, namely $\pm 2.5 \mathrm{~K}$ for the $\mathrm{Tb}$ and $\pm 3 \mathrm{~K}$ for the SST. Using the full database for December 1999, the retrieval yields $3.15 \pm 1.38 \mathrm{~mm} \mathrm{~h}^{-1}$, having found 10152 database entries that match the observation vector within the specified uncertainty. The retrieval uncertainty is $44 \%$. If database completeness errors of less than $1 \%$ are sought for this pixel, then approximately 2000 (i.e., $44^{2}$ ) database entries fitting the above observations are required in this example. At the $10 \%$ level, only 20 observations are needed. Results from a retrieval using subsets of the complete database are shown in Table 2 to confirm these predictions.

The mean and standard deviation of the retrieved rainfall begin to deviate by approximately $1 \%$ as the number of database entries fitting the observations decreases to 2550 in agreement with the predictions. Errors increase to $10 \%$ for somewhat fewer than 40 database entries, also in general agreement with predictions.

Database completeness errors are well below the 1\% threshold for the algorithm presented here. They could

TABLE 2. Sensitivity of retrieved rainfall as a function of database entries matching a set of observations.

\begin{tabular}{ccc}
\hline & $\begin{array}{c}\text { Matching entries } \\
(\mathrm{Tb}=30 \pm 2.5 \mathrm{~K} ; \\
\begin{array}{c}\text { Database } \\
\text { entries }\end{array}\end{array}$ & $\begin{array}{c}\text { Rainfall } \\
\left(\mathrm{mm} \mathrm{h}^{-1}\right)\end{array}$ \\
\hline 666713 & 10.152 & $3.15 \pm 1.38$ \\
333356 & 5128 & $3.15 \pm 1.38$ \\
166679 & 2550 & $3.15 \pm 1.41$ \\
83339 & 1256 & $3.17 \pm 1.38$ \\
41669 & 605 & $3.13 \pm 1.41$ \\
20834 & 294 & $3.09 \pm 1.49$ \\
10417 & 148 & $3.11 \pm 1.45$ \\
5208 & 86 & $3.23 \pm 1.68$ \\
2604 & 43 & $3.42 \pm 2.03$ \\
1302 & 22 & $3.86 \pm 2.67$ \\
\hline
\end{tabular}


a

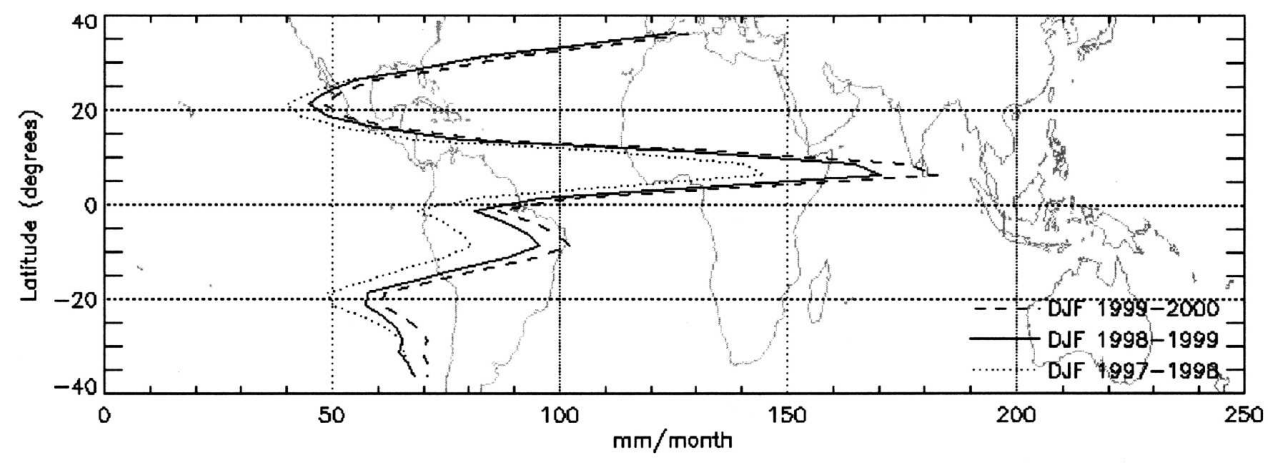

b

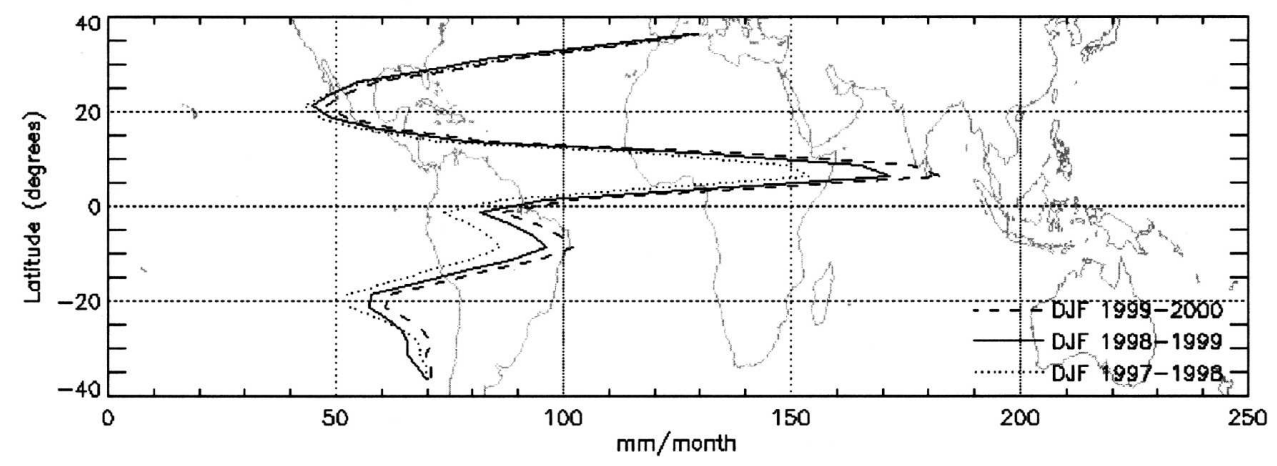

FIG. 5. Zonal mean rainfall accumulation for Dec 1999 using three distinct databases created for DJF 1997-98, 1998-99, and 1999-2000. (a) Uses the corresponding rain/no-rain lookup tables created for each of the time periods; (b) uses the rain/no-rain lookup table from DJF 1999-2000 for all three retrievals.

become important, however, if the algorithm is modified to include additional constraints. In addition, there is the possibility that the TRMM PR solutions used to construct the a priori database do not fully cover the range of possible solutions. Because the lower threshold of the TRMM PR $\left(0.5 \mathrm{~mm} \mathrm{~h}^{-1}\right)$ is very low when averaged over the much larger radiometer FOV, and the upper threshold of the TRMM PR (when the echo is completely attenuated) has not occurred in the TRMM data, these issues were considered unimportant in this study. In the next section, an additional spatial constraint is added to test the algorithm's sensitivity to spatial variations in the a priori database. Additional constraints can also be added if additional Tbs, or geophysical parameters, such as the wind speed or freezing level, are added as part of the observation vector.

\section{c. Space/time dependence of the a priori database}

Random errors related to the inversion can be reduced by standard statistical methods when space and time averages are considered and errors are assumed uncorrelated. Errors, however, are not uncorrelated.
Indeed, Fig. 1 clearly identifies temporal biases across the Pacific that could not exist if all errors were assumed random. Instead, Fig. 1 suggests that cloud properties change systematically across the Pacific basin as a function of still uncertain regime changes.

The sensitivity of the error algorithm to regional and temporal changes in cloud properties can be investigated by constructing independent databases for separate time periods and locations. Ideally, the retrieval would be insensitive to the details of when and where the database was created as long as it has enough entries to represent a stable solution. The first experiment, therefore, is to compare the rainfall retrieved for December 1999 from three distinct databases corresponding to DJF 1997-98, 1998-99, and 1999-2000, respectively. Because each database is associated with a rain probability lookup table from its own time period, the sensitivity of the retrieval to changes in these tables is also examined. Figure 5a shows zonal mean rainfall accumulations using the three distinct databases with their corresponding rain probability tables. The El Niño database (DJF 1997-98) yields only $74.9 \mathrm{~mm}$ 
month $^{-1}$ for December 1999, while the La Niña database yields $83.0 \mathrm{~mm}$ and the reference database (DJF 1999-2000) yields $88.7 \mathrm{~mm}$ as before. Based upon this very limited comparison, the retrieval algorithm can be assigned an uncertainty on the order of $15 \%$ in the global rainfall resulting from interannual variability of the cloud population. Figure 5b shows the equivalent results, but using the DJF 1999-2000 rain probability lookup table in conjunction with each of the three rain databases. Results here are somewhat better. The El Niño database now yields $79.2 \mathrm{~mm}$, while the La Niña database yields $83.6 \mathrm{~mm}$. The reference is unchanged by this experiment. This represents roughly a $5 \%$ reduction in the uncertainty. From this, we estimate that approximately $1 / 3$ or $5 \%$ of the overall uncertainty is a result of changes in the rainfall probability table; the remaining $2 / 3$ or $10 \%$ are a result of changes in the rain databases themselves.

To test the algorithm sensitivity to spatially subsetted databases, the retrieval algorithm is modified slightly. For this study, additional weight is given to pixels based upon the physical distance between the location of a pixel and the location of the database entries that fit the observations. A Gaussian function with a half-width of $2000 \mathrm{~km}$ is used to ensure that instabilities are not introduced because of insufficient database entries. The retrieved rainfall accumulation for December 1999 using the entire DJF 1999-2000 database is $2.77 \mathrm{~mm}$ day $^{-1}$, the regionally constrained retrieval yields 2.68 mm day ${ }^{-1}$. The accumulation maps appear quite similar and are therefore not shown. To highlight the regional differences, Fig. 6 directly compares the $2.5^{\circ} \times 2.5^{\circ}$ accumulations from the two methods. Overall, uncertainties resulting from regional variations in the a priori database lead to approximately $10 \%$ uncertainty in the monthly accumulation when compared at a $2.5^{\circ}$ grid scale.

Differences of the order of $10 \%-20 \%$ related to spatial and temporal changes in cloud properties are not limited to the error algorithm described here. Differences of this magnitude and larger are also clearly evident in many of the products compared as part of the third Precipitation Intercomparison Project (PIP-3) presented by Adler et al. (2001). In the case of the error algorithm, however, the source of the regional and temporal variations can be traced directly to the algorithm formulation. The variable terms in Eq. (1), while not explicitly enumerated earlier, are related to differences in TRMM PR-derived rainfall characteristics within each TMI FOV. These variables are denoted here as "database variables." Their seasonal and temporal variations must be responsible for regional and temporal differences in the output product, because all other

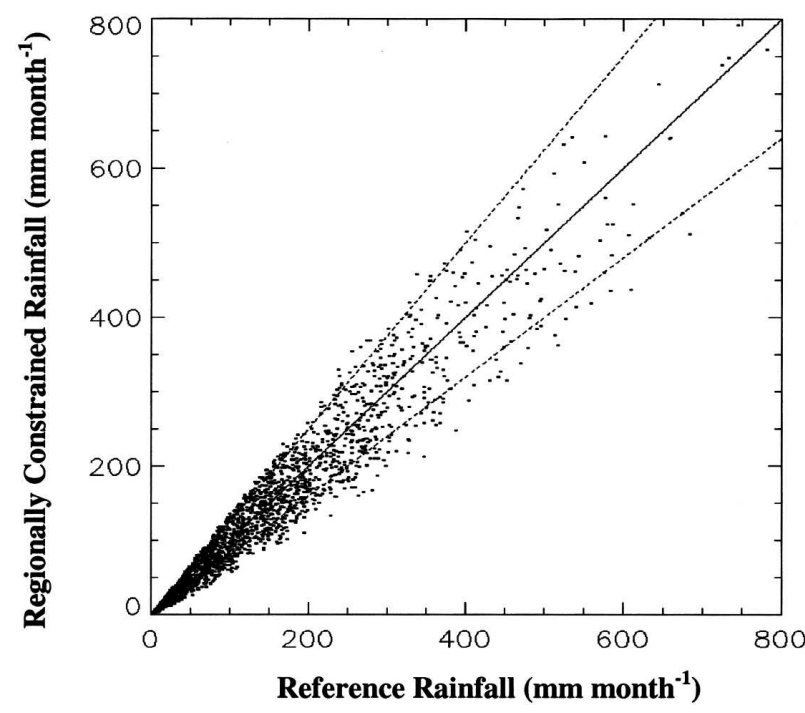

FIG. 6. Comparison of the retrieved rainfall accumulations for Dec 1999, using the complete DJF 1997-2000 database vs the same database but with additional regional constraints. Differences between the two products represent $2.5^{\circ}$ areas.

parameters were specifically held constant in the formulation of the error algorithm.

Variations of TRMM PR-derived rainfall within a single 19-GHz FOV cannot fully be described with only a few parameters because details of the vertical profile and the relative position of individual TRMM PR pixels within the larger TMI FOV both affect the upwelling TMI radiances. Nonetheless, it may be reasonable to assume that variation can be represented by 1) the mean FL, defined as the mean height of the layer above the bright band; 2) the rainfall inhomogeneity $\left(\sigma_{R}\right)$, computed as the standard deviation of the TRMM PR rain pixels with the $3-\mathrm{dB}$ gain of the TMI FOV divided by the mean rainfall in the TMI FOV; 3) the mean slope (slope) of the rainfall profile within the radiometer FOV as computed by finding the best-fit line passing through the surface rainfall to the top of the rain only layer; and 4) the surface wind speed. These four parameters represent the database variables and were added to the a priori database during its construction. Systematic changes in these properties, which the current algorithm does not independently retrieve, can then be hypothesized to be responsible for the regional and seasonal changes in the output product.

An illustrative example is used to verify this assertion. A Tb difference of $30 \mathrm{~K}$ with an SST of $300 \mathrm{~K}$ is used. For this example, the FL is assumed to be $4500 \pm$ $200 \mathrm{~m}$, the slope is $-10 \pm 10 \% \mathrm{~km}^{-1}$, and the rainfall variability is assumed to be $3 \pm 0.5$. In general, wind statistics do not change much from one region to another at monthly time scales. As such, surface wind 
TABLE 3. Difference (\%) between mean $2.5^{\circ}$ rain retrieval using a global vs a regional database as a function of additional constraints added to the retrieval. "Tropical" refers to latitudes between $15^{\circ}$, while "extratropical" refers to latitudes between $25^{\circ}$ and $30^{\circ}$.

\begin{tabular}{|c|c|c|c|c|c|}
\hline \multicolumn{3}{|c|}{ Constraints } & \multirow[b]{2}{*}{ Global } & \multirow[b]{2}{*}{ Tropical } & \multirow[b]{2}{*}{ Extratropical } \\
\hline Variability & Slope & FL & & & \\
\hline- & - & - & $12.0 \%$ & $6.5 \%$ & $19.2 \%$ \\
\hline Yes & - & - & $6.8 \%$ & $4.2 \%$ & $10.3 \%$ \\
\hline- & Yes & - & $10.7 \%$ & $5.9 \%$ & $17.3 \%$ \\
\hline- & - & Yes & $4.0 \%$ & $3.6 \%$ & $4.5 \%$ \\
\hline Yes & Yes & - & $6.3 \%$ & $4.2 \%$ & $9.3 \%$ \\
\hline Yes & - & Yes & $2.4 \%$ & $2.2 \%$ & $2.5 \%$ \\
\hline- & Yes & Yes & $3.5 \%$ & $3.2 \%$ & $3.8 \%$ \\
\hline Yes & Yes & Yes & $2.4 \%$ & $2.2 \%$ & $2.2 \%$ \\
\hline
\end{tabular}

speed is unlikely to cause regional and temporal biases at these time scales. Unconstraining the wind speed did not cause errors in excess of $1 \%$ for any of the simulations performed. As such, wind is not included to ensure that sufficient database entries were available to obtain robust statistics. The retrieval is run first using the three DJF databases without additional information related to the database variables. The unconstrained retrieval yields $2.62 \pm 1.31,3.00 \pm 1.36$, and $3.15 \pm 1.39$ $\mathrm{mm} \mathrm{h}^{-1}$ for databases constructed from DJF 1997-98, 1998-99, and 1999-2000, respectively. When the retrieval is constrained by the three additional database variables presented above, results are $3.21 \pm 0.74,3.26$ \pm 0.67 , and $3.23 \pm 0.71 \mathrm{~mm} \mathrm{~h}^{-1}$ for the three databases, respectively. The constrained retrieval is seen to be very consistent for the three databases, reducing the interannual variability from approximately $15 \%$ for the unconstrained retrieval to a mere $1 \%-2 \%$ when the database variables are explicitly supplied to the algorithm. Random errors are also smaller as would be expected for a more highly constrained inversion. As seen in the previous section, however, a larger reference database is also required if stability is to be guaranteed at the $1 \%$ level.

The above experiment can also be performed to assess regional variations in the constrained and unconstrained retrievals. For this experiment, the same geographical constraint as before is used, but $2.5^{\circ}$ monthly accumulations are compared as each database variable and combination of variables are constrained. The retrieval is run only for TMI pixels that have coincident radar data, and the constraint is taken directly from TRMM PR observations. Results are presented in Table 3 for all pixels as well as tropical $\left(-15^{\circ}<\right.$ lat $<$ $15^{\circ}$ ) and extratropical (|lat $>25^{\circ}$ ) pixels.

Global results show a mean difference of $12 \%$ for the unconstrained retrieval. This is consistent with results shown in Fig. 6. Of the three database variables, knowing the FL is seen to have the greatest impactreducing the mean uncertainty to $4.0 \%$. The spatial variability alone reduces the uncertainty to $7 \%$, while knowledge of the slope of the rainfall profile seems to reduce errors only marginally. Combinations of variables show consistent trends. Knowledge of the FL and rainfall variability reduces errors to $2.4 \%$. Adding information on the slope fails to further reduce the uncertainty. In all of these experiments, the effect of the rain/no-rain database could be reduced to approximately $1 \%$ if SST is assumed known to $1 \mathrm{~K}$.

For the tropical subset, the overall uncertainty is reduced by a factor of 2 , but the relative contributions of the three database variables are quite similar to the global results. For the extratropical subset the unknown FL is seen to be the dominant source of uncertainty. This is consistent with the expectation that freezing levels are much more variable in the extratropics than they are in the Tropics.

\section{d. Uncertainties resulting from algorithm formulation}

Up to this point, the errors have focused on uncertainties introduced by inversion methodology as well as errors in the TRMM PR-derived rainfall, database completeness issues, and regional and seasonal changes in the formulation variables. No mention has been made of errors in the algorithm formulation itself. This could consist of a poor formulation of any of the assumptions detailed in section 2. Clearly, there is evidence for excess extinction in the bright band (e.g., Bauer et al. 2000; Olson et al. 2001; Battaglia et al. 2003) that represents a potential error of omission in the current formulation. Yet, even a perfect reformulation of the brightband extinction would do nothing to reduce the uncertainties as examined in any of the previous sections. These additional errors, termed algorithm formulation errors, are included in both the database and inversion, and must therefore be quantified using independent measurements.

Traditional validation efforts have generally been limited to long-term comparisons between satellite- and ground-based rainfall. Over a sufficiently long time, this procedure eliminates the random errors from the comparison. Done indiscriminately, however, such procedures ignore the effect of the database variables, which can be responsible for $10 \%-15 \%$ regional $/$ seasonal biases explored in the previous section. Instead, it seems necessary to adopt a physical validation paradigm that explicitly accounts for the cloud properties at the particular validation site. In this latter paradigm, a critical 
first step is to correctly identify and account for all the database variables.

In the case of the simple error algorithm presented here, the database variables can all be observed directly by TRMM PR. They can, however, also be observed at well-designed validation sites and added to the retrieval as additional constraints similar to what was done in the previous section. Using the validation site to observe the database variables allows comparisons to be made for any microwave radiometer instead of just TMI over the narrow swath that overlaps the TRMM PR sensor. The constrained algorithm, run at a small number of validation sites, should show only the $2 \%-3 \%$ residual regional and temporal variability found in the previous section. Biases beyond 3\%, used to denote the accuracy of the constrained retrieval, must then be ascribed either to biases in the PR-derived rainfall, as outlined in section $3 \mathrm{a}$, or to incorrect values in the constant terms defined in section 2. If the errors exceed the possible errors introduced by overall PR biases, then the errors are labeled as formulation errors.

Because the final error model must include possible formulation errors, the outlined validation activity is seen as a critical component to this effort. Without it, only those variables explicitly included in the database can be accounted for and the error model loses its quantitative foundation. Accomplishing this task requires a flexible validation strategy that can measure the surface rainfall, verify that the uncertainty in the input data ( $\mathrm{Tb}$ and SST in the present case) are correctly specified, measure database variables, and observe the constant parameters to see whether residual errors are larger than the predicted values. While such an activity is well beyond the scope of this paper, it is highlighted in order to stress that a full error model must indeed account for all sources of error and not merely those deemed tractable in the algorithm formulation.

\section{Summary and conclusions}

A very simple microwave radiometer algorithm using only the $\mathrm{Tb}$ difference at $19 \mathrm{GHz}\left(\mathrm{Tb}=\mathrm{Tb}_{19 \mathrm{~V}}-\right.$ $\left.\mathrm{Tb}_{19 \mathrm{H}}\right)$ and the SST was developed in order to establish a framework for end-to-end error modeling. In addition to the inversion uncertainty, three sources of uncertainty were identified related to the a priori database used by the retrieval algorithm. The first is the result of uncertainties in the TRMM PR rainfall used to establish the a priori database $\varepsilon_{\text {database }}$. Results indicated that the rain information in the radiometer brightness temperatures could reduce errors in the TRMM PRderived rainfall used in the a priori database by a factor of 4 , or approximately $5 \%$, if the TRMM PR is assumed to be uncertain at the $20 \%$ level. A more transparent TRMM PR rain algorithm that directly couples rain intensity to the shape of the rain profile through uncertainties in the DSD assumption was discussed as a possible method to verify this result more rigorously.

The second source of uncertainty was related to database completeness issues. It was shown that the algorithm, as formulated for global retrievals, should have no discernable errors resulting from the database size if the reference database is used. The completeness error $\varepsilon_{\text {complete }}$ can safely be set to zero in the case that each DJF database contains approximately 600000 entries. If the algorithm is modified to include additional input data, or the uncertainty in the input data is reduced, the completeness issues can be reexamined considering both the inversion uncertainty as well as the number of entries found in the database for any specific set of input parameters.

The third source of errors was related to the temporal and regional variations in the a priori database that were not resolved by the retrieval algorithm. These errors were defined as $\varepsilon_{\text {space/time }}$, and were found to be approximately $15 \%$ for temporal variations and $10 \%$ for regional variations examined at $2.5^{\circ}$ resolution. Because the temporal variations were quantified using DJF for an El Niño, a La Niña, and a weak La Niña year, the seasonal variability is likely to represent an upper bound. The regional variability, examined using a composite DJF 1997-2000 database showed an additional $10 \%$ uncertainty. Together, these two terms may be estimated to contribute $25 \%$ uncertainty to the overall solution. It was shown that better constraints on SST, rain variability, and FL could be used to reduce this number quite significantly. Algorithms developed specifically to retrieve the FL, such as that developed by Wilheit et al. (1991), could be used to reduce the current uncertainty.

Finally, possible algorithm formulation errors were considered. These errors $\varepsilon_{\text {formulation }}$ were not quantified here because they required independent validation data for which the TRMM PR sensor cannot be used. Instead, a physical validation paradigm was outlined that explicitly accounts for the database variables before averaging satellite and ground observations.

As long as the error sources are assumed independent, it is relatively straightforward to sum the different sources into a final uncertainty,

$$
\begin{aligned}
\varepsilon_{\text {total }}= & {\left[\left(N^{-1 / 2} \varepsilon_{\text {inversion }}\right)^{2}+\left(\varepsilon_{\text {correctness }}\right)^{2}+\left(\varepsilon_{\text {concept }}\right)^{2}\right.} \\
& \left.+\left(\varepsilon_{\text {space/time }}\right)^{2}+\left(\varepsilon_{\text {formulation }}\right)^{2}\right]^{0.5}
\end{aligned}
$$


where $N$ is the number of independent samples used in any rain accumulation. Clearly, the inversion errors will dominate any uncertainties at the pixel level. Unless the formulation errors turn out to be comparable to the random errors, the latter may be used as a good estimate of the total error. At time and space scales that are important for climate studies, the number of independent pixels $N$ becomes large and the inversion errors disappear. If the algorithm is again assumed to have no formulation errors, then the measurement uncertainty for monthly rain accumulations over $2.5^{\circ}$ areas can be estimated from $\varepsilon_{\text {correctness }}=5 \%$ and $\varepsilon_{\text {space/time }}=25 \%$. This yields a total error of $\varepsilon_{\text {total }}=$ $26 \%$, which is of course very large for uncertainties related to climate scales. The large value, however, is perhaps simply a reflection of the simple algorithm used here. At intermediate space and time scales, additional information regarding the time and space correlations of the variable parameters will be necessary to estimate the true number of independent samples. These, and already mentioned issues and complexities raised in the paper, however, make it clear that significant additional effort will be required before error modeling achieves the same maturity as retrieval algorithms have. This work is ongoing.

Acknowledgments. The authors wish to thank the many individuals who have provided continuous feedback as the ideas presented here have evolved. In particular, we thank Drs. Thomas Wilheit and Toshio Iguchi for many discussions on the details related to error modeling physical validation. This work was supported by NASA through TRMM Grant NAG5-13694 and AMSR Grant NNG-04 H245G. Sea surface wind speed and SST data were produced by Remote Sensing Systems and sponsored by the NASA Earth Science REASoN DISCOVER project.

\section{REFERENCES}

Adler, R. F., C. Kidd, G. Petty, M. Morissey, and M. H. Goodman, 2001: Intercomparison of global precipitation products: The third Precipitation Intercomparison Project (PIP-3). Bull. Amer. Meteor. Soc., 82, 1377-1396.

Aonashi, K., and G. Liu, 2000: Passive microwave precipitation retrievals using TMI during the Baiu period of 1998. Part I: Algorithm description and validation. J. Appl. Meteor., 39, 2024-2037.

Battaglia, A., C. Kummerow, D.-B. Shin, and C. Williams, 2003: Constraining microwave brightness temperatures by radar brightband observations. J. Atmos. Oceanic Technol., 20, 856-871.

Bauer, P., 2001: Over-ocean rainfall retrieval from multisensor data of the Tropical Rainfall Measuring Mission. Part I: Design and evaluation of inversion databases. J. Atmos. Oceanic Technol., 18, 1315-1330.
—, A. Khain, A. Pokrovsky, R. Meneghini, C. Kummerow, F. Marzano, and J. P. V. Poiares Baptista, 2000: Combined cloud-microwave radiative transfer modeling of stratiform rainfall. J. Atmos. Sci., 57, 1082-1104.

Berg, W., C. Kummerow, and C. A. Morales, 2002: Differences between east and west Pacific rainfall systems. J. Climate, 15, 3659-3672.

Chang, A. T. C., L. S. Chiu, C. Kummerow, and J. Meng, 1999: First results of the TRMM microwave imager (TMI) monthly oceanic rain rate: Comparison with SSM/I. Geophys. Res. Lett., 26, 2379-2382.

Hong, Y., T. T. Wilheit, and W. R. Russell, 1997: Estimation of monthly rainfall over oceans from truncated rain-rate samples: Applications to SSM/I data. J. Atmos. Oceanic Technol., 14, 1012-1022.

Iguchi, T., T. Kozu, R. Meneghini, J. Awaka, and K. Okamoto, 2000: Rain-profiling algorithm for the TRMM precipitation radar. J. Appl. Meteor., 39, 2038-2052.

Kiehl, J. T., and K. E. Trenberth, 1997: Earth's annual global mean energy budget. Bull. Amer. Meteor. Soc., 78, 197-208.

Kummerow, C., 1993: Radiative transfer model comparisons for passive microwave frequencies. J. Geophys. Res., 98, 27572765.

_ , and L. Giglio, 1994: A passive microwave technique for estimating rainfall and vertical structure information from space. Part I: Algorithm description. J. Appl. Meteor., 33, 3-18.

- and Coauthors, 2001: The evolution of the Goddard profiling algorithm (GPROF) for rainfall estimation from passive microwave sensors. J. Appl. Meteor., 40, 1801-1820.

L'Ecuyer, T. S., and G. L. Stephens, 2002: An uncertainty model for Bayesian Monte Carlo retrieval algorithms: Application to the TRMM observing system. Quart. J. Roy. Meteor. Soc., 128, 1713-1737.

Marzano, F. S., A. Mugnai, G. Panegrossi, N. Pierdicca, E. A. Smith, and J. Turk, 1999: Bayesian estimation of precipitating cloud parameters from combined measurements of spaceborne microwave radiometer and radar. IEEE Trans. Geosci. Remote Sens., 37, 596-613.

Masunaga, H., T. Iguchi, R. Oki, and M. Kachi, 2002: Comparison of rainfall products derived from TRMM microwave imager and precipitation radar. J. Appl. Meteor., 41, 849-862.

Mugnai, A., E. A. Smith, and G. J. Tripoli, 1993: Foundations for statistical-physical precipitation retrieval from passive microwave satellite measurements. Part II: Emission-source and generalized weighting-function properties of a timedependent cloud-radiation model. J. Appl. Meteor., 32, 17-39.

Olson, W. S., P. Bauer, C. D. Kummerow, Y. Hong, and W.-K. Tao, 2001: A melting-layer model for passive/active microwave remote sensing applications. Part II: Simulation of TRMM observations. J. Appl. Meteor., 40, 1164-1179.

Panegrossi, G., and Coauthors, 1998: Use of cloud model microphysics for passive microwave-based precipitation retrieval: Significance of consistency between model and measurement manifolds. J. Atmos. Sci., 55, 1644-1673.

Petty, G. W., 1994: Physical retrievals of over-ocean rain rate from multichannel microwave imagery. Part I: Theoretical characteristics of normalized polarization and scattering indices. Meteor. Atmos. Phys., 54, 79-99.

Roberti, L., J. Haferman, and C. Kummerow, 1994: Microwave radiative transfer through horizontally inhomogeneous precipitating clouds. J. Geophys. Res., 99, 16 707-16 718.

Robertson, F. R., D. E. Fitzjarrald, and C. D. Kummerow, 2003: 
Effects of uncertainty in TRMM precipitation radar path integrated attenuation on interannual variation of tropical oceanic rainfall. Geophys. Res. Lett., 30, 1180, doi:10.1029/ 2002 GL016416.

Smith, E. A., X. Xiang, A. Mugnai, and G. Tripoli, 1994: Design of an inversion-based precipitation profile retrieval algorithm using an explicit cloud model for initial guess microphysics. Meteor. Atmos. Phys., 54, 53-78.

, P. Bauer, F. S. Marzano, C. D. Kummerow, D. McKague, A.
Mugnai, and G. Panegrossi, 2002: Intercomparison of microwave radiative transfer models for precipitating clouds. IEEE Trans. Geosci. Remote Sens., 40, 541-549.

Wilheit, T. T., 1986: Some comments on passive microwave measurement of rain. Bull. Amer. Meteor. Soc., 67, 1226-1232. , A. T. C. Chang, and L. S. Chiu, 1991: Retrieval of monthly rainfall indices from microwave radiometric measurement using probability distribution functions. J. Atmos. Oceanic Technol., 8, 118-136. 